\section{Mehr Strikturrezidive mit neuer Maßeinheit erkennen}

\author{
Die Differenz zwischen maximalem und durchschnittlichem Harnfluss hilft, \\ postoperative Rezidive von Harnröhrenstrikturen ohne Zystoskopie zu \\ erkennen.
}

$\mathrm{D}$ ie Uroflowmetrie ist eine einfache Methode, die Entleerungsfunktion der Harnblase nicht invasiv zu testen. Speziell wird sie bei Kontrollen von $\mathrm{Pa}$ tienten eingesetzt, bei denen eine Harnröhrenstriktur mithilfe einer Urethraplastik beseitigt worden ist. Als möglicher Indikator für Strikturrezidive gelten maximale Flowraten $(\mathrm{Qm})$ von unter $10-15 \mathrm{ml} / \mathrm{s}$.

Urologen vom Trauma and Urologic Reconstruction Network for Surgeons haben nun in einer prospektiven Studie untersucht, wie verlässlich die Flowmessung in der Rezidivdiagnostik ist. Das Vorliegen oder Nichtvorliegen von Strikturen wurde zystoskopisch gesichert. Knapp 1.200 Männer mit operativ behandelter Harnröhrenstriktur waren an der Studie beteiligt. 59 \% der Männer mit nachweislicher erneuter Striktur hatten einen maximalen Flow von mehr als $15 \mathrm{ml} / \mathrm{s}$, $79 \%$ einen Qm-Wert von $\geq 10 \mathrm{ml} / \mathrm{s}$. Besser sah das Ergebnis aus, wenn die Differenz zwischen maximalem und durchschnittlichem Harnfluss (Qm-Qd) errechnet wurde. Differenzen von $10 \mathrm{ml} / \mathrm{s}$ und mehr wiesen nur $17 \%$ der Männer mit Rezidiv einer Urethrastriktur auf. Für einen postoperativen Qm von $<10 \mathrm{ml} / \mathrm{s}$ ergab sich eine Sensitivität von $21 \%$, Spezifität von $97 \%$, positiver Vorhersagewert von $60 \%$ und negativer Vorhersagewert von $85 \%$. Eine Schwelle von $15 \mathrm{ml} / \mathrm{s}$ erbrachte Werte von $41 \%, 88 \%, 43 \%$ und $87 \%$. Bei einer Schwelle von $10 \mathrm{ml} / \mathrm{s}$ führte $\mathrm{Qm}-\mathrm{Qd}$ zu einer Sensitivität von $83 \%$, einer Spezi- fität von $58 \%$, einem positiven Vorhersagewert von $30 \%$ und einem negativen Vorhersagewert von $94 \%$. Speziell bei Männern bis 40 Jahren erwies sich die Flowdiagnostik als hilfreich.

Fazit: „Qm allein ist möglicherweise nicht sensitiv genug, um die Zystoskopie in der Rezidivdiagnostik von Harnröhrenstrikturen zu ersetzen“, resümieren die Autoren. Besser werde die Entleerungskurve durch Qm-Qd repräsentiert. Allerdings geht dessen höhere Sensitivität mit deutlich niedrigerer Spezifität einher. Von 100 Männern ohne Rezidiv würden mit einem Qm-QdSchwellenwert von $10 \mathrm{ml} / \mathrm{s} 42$ fälschlich als strikturpositiv diagnostiziert. Zwar ist $\mathrm{Qm}-\mathrm{Qd}$ in der Gesamtsicht Qm überlegen. Das optimale Mittel, den Patienten unnötige Zystoskopien zu ersparen, scheint damit dennoch nicht gefunden zu sein.

Dr. Robert Bublak

Tam CA et al. Critical analysis of the use of uroflowmetry for urethral stricture disease surveillance. Urology. 2016;

doi:10.1016/j.urology.2015.12.070.

\section{Verdacht auf Nierenkrebs: Biopsie verlässlich?}

\author{
Ist auf Biopsien unklarer Raumforderungen der Niere Verlass? Die Antwort \\ erinnert an die Auskünfte, die früher von Radio Eriwan her bekannt waren: \\ Im Prinzip ja, aber ...
}

D ie deutsche S3-Leitlinie Diagnostik, Therapie und Nachsorge des Nierenzellkarzinoms erklärt zur bioptischen Sicherung von Nierentumoren: „Die Biopsie einer unklaren Raumforderung der Niere sollte nur erfolgen, wenn dies die Therapiewahl beeinflussen könnte." Dies wäre beispielsweise bei der Selektion von Patienten mit kleinen Tumoren der Fall, bei denen eine Operation der Niere vermieden werden soll und/oder eine Radiofrequenzablation respektive eine Kryoablation geplant ist.

Ein Team um Hiten Patel, Johns Hopkins Medical Institutions, Baltimore, hat 20 einschlägige Studien gesichtet, die sich mit Nierenbiopsien bei Verdacht auf ein lokalisiertes Nierenzellkarzinom beschäftigt hatten. 2.979 Patienten mit
3.113 Biopsien waren beteiligt. Überwiegend war es dabei um Stanzbiopsien gegangen, nur in einer Studie hatte man die Feinnadelaspiration untersucht.

Auf den ersten Blick lieferten die Stanzen ordentliche Resultate, die Spezifität erreichte $96,2 \%$, die Sensitivität lag bei $97,5 \%$. Das führte zu einem positiven Vorhersagewert von 99,8\%. Das Aber stellte sich beim Blick auf den negativen Vorhersagewert ein, der selbst in den Studien mit dem geringsten Verzerrungspotenzial bei nur 72,7 \% lag. Damit entpuppte sich die fragliche Raumforderung bei mehr als jedem vierten Patienten mit bioptisch negativem Befund hinterher doch als Nierenzellkarzinom. $16 \%$ der laut Biopsiediagnose prognostisch günstigen Malignome (Fuhrman-Grad
1-2) mussten in der postoperativen $\mathrm{Pa}$ thologie als ungünstig eingestuft werden (Grad 3-4). Etwa jede siebte Stanze lieferte nicht ausreichend Material für eine Diagnose - gut $90 \%$ der Patienten, die nach einer solchen nicht diagnostischen Biopsie operiert wurden, hatten Nierenkrebs.

Fazit: „Aus den vorhandenen Nachweisen zu schließen, die Biopsie renaler Raumforderungen sei eine allgemeine Voraussetzung für die klinische Entscheidungsfindung, ist nicht statthaft", schreiben Patel et al. Die diagnostische Genauigkeit der Gewebeentnahmen aus lokalen renalen Raumforderungen sei generell hoch, doch die Rate der nicht diagnostischen Biopsien und der negative Vorhersagewert seien bedenklich.

Dr. Robert Bublak

Patel HD et al. Diagnostic accuracy and risks of biopsy in the diagnosis of a renal mass suspicious for localized renal cell carcinoma: systematic review of the literature. J Urol. 2016; doi:10.1016/j.juro.2015.11.029. 